\title{
Jackiw-Pi Model: A Superfield Approach ${ }^{1}$
}

\author{
Saurabh Gupta \\ The Institute of Mathematical Sciences, CIT Campus, Chennai - 600 113, India \\ e-mails: saurabh@imsc.res.in, guptasaurabh4u@gmail.com
}

\begin{abstract}
We derive the off-shell nilpotent and absolutely anticommuting Becchi-RouetStora-Tyutin (BRST) as well as anti-BRST transformations $s_{(a) b}$ corresponding to the YangMills gauge transformations of 3D Jackiw-Pi model by exploiting the "augmented" superfield formalism. We also show that the Curci-Ferrari restriction, which is a hallmark of any non-Abelian 1-form gauge theories, emerges naturally within this formalism and plays an instrumental role in providing the proof of absolute anticommutativity of $s_{(a) b}$.
\end{abstract}

PACS : 11.15.-q, 03.70.+k, 11.10.Kk, 12.90.+b

Keywords: Jackiw-Pi model; augmented superfield formalism; Curci-Ferrari restriction

\section{Introduction}

Standard Model (SM) of particle physics accounts for three out of four fundamental interactions of nature. In spite of the stunning success of SM, which is based on the non-Abelian 1-form gauge theories, one of the main issues with gauge theories are connected with the co-existence of mass and gauge invariance together. However, the gauge invariance does not necessarily imply the masslessness of gauge particles for sufficiently strong vector couplings [1. In this context, it is worth mentioning about the models where 1-form gauge field acquires a mass in a natural fashion such as $4 \mathrm{D}$ topologically massive (non-)Abelian gauge theories (with $B \wedge F$ term) [2, 3, 4]. But, these models suffer from the problems related with renormalizability, consistency and unitarity.

Furthermore, massive gauge theories in other than $(3+1)$-dimensions of spacetime, which are free from the problems of $4 \mathrm{D}$ topologically massive models, have been studied for a quite some time (see, e.g. [5]). The $(2+1)$-dimensional Jackiw-Pi (JP) model is one such model where mass and gauge-invariance exist together. The JP model is a parity even model and endowed with two sets of local continuous symmetries, namely; the usual Yang-Mills (YM) and non-Yang-Mills (NYM) symmetries. This model has been studied throughly (see, e.g. [6, 7, 8, 9]).

In this write-up, we have applied "augmented" superfield approach to BRST formalism in order to derive the off-shell nilpotent and absolutely anticommunting (anti-)BRST symmetry transformations corresponding to the YM gauge symmetry transformations of JP model. The anticommutativity of (anti-)BRST symmetry transformations is ensured by the CurciFerrari (CF) restriction which emerges naturally in this framework. We would like to point out that, within the framework of superfield formalism, a general set up for BRST analysis of a general gauge system also exists [10]. Our present analysis could be thought of as an application of this approach to a specific model having a closed gauge algebra.

\footnotetext{
${ }^{1}$ Talk delivered at BLTP, JINR, Dubna (Moscow Region), Russia in the International Workshop on "Supersymmetries \& Quantum Symmetries"(SQS'2013) during July 29 - August 3, 2013.
} 


\section{Jackiw-Pi Model: Symmetries}

The Lagrangian density of $(2+1)$-dimensional Jackiw-Pi model2 is given as follows [7, 8]:

$$
\mathcal{L}=-\frac{1}{4} F^{\mu \nu} \cdot F_{\mu \nu}-\frac{1}{4}\left(G^{\mu \nu}+g F^{\mu \nu} \times \rho\right) \cdot\left(G_{\mu \nu}+g F_{\mu \nu} \times \rho\right)+\frac{m}{2} \varepsilon^{\mu \nu \eta} F_{\mu \nu} \cdot \phi_{\eta},
$$

where $F_{\mu \nu}=\partial_{\mu} A_{\nu}-\partial_{\nu} A_{\mu}-g\left(A_{\mu} \times A_{\nu}\right)$ and $G_{\mu \nu}=D_{\mu} \phi_{\nu}-D_{\nu} \phi_{\mu}$ are 2-form curvature tensors corresponding to the 1 -form fields $A_{\mu}$ and $\phi_{\mu}$, respectively. Moreover, $\rho$ is a scalar field and $m$ represents the mass parameter. In the above, $A_{\mu}$ and $\phi_{\mu}$ have opposite parity which makes JP model to be a parity conserving model.

The above Lagrangian density (1) respects two sets of local symmetry transformations, the YM gauge transformations $\left(\delta_{1}\right)$ and NYM gauge transformations $\left(\delta_{2}\right)$, namely [7, 8];

$$
\begin{gathered}
\delta_{1} A_{\mu}=D_{\mu} \Lambda, \quad \delta_{1} \phi_{\mu}=-g\left(\phi_{\mu} \times \Lambda\right), \quad \delta_{1} \rho=-g(\rho \times \Lambda), \\
\delta_{2} A_{\mu}=0, \quad \delta_{2} \phi_{\mu}=D_{\mu} \Sigma, \quad \delta_{2} \rho=+\Sigma, \quad \delta_{2} F_{\mu \nu}=0,
\end{gathered}
$$

where $\Lambda=\Lambda \cdot T$ and $\Sigma=\Sigma \cdot T$ are $S U(N)$ valued infinitesimal gauge parameters corresponding to YM and NYM gauge transformations, respectively. It is straightforward to check that $\delta_{1}$ and $\delta_{2}$ are the symmetry transformations, as: $\delta_{1} \mathcal{L}=0, \quad \delta_{2} \mathcal{L}=\partial_{\mu}\left[\frac{m}{2} \varepsilon^{\mu \nu \eta} F_{\nu \eta} \cdot \Sigma\right]$.

\section{Augmented Superfield Approach: A Synopsis}

We apply Bonora-Tonin's superfield formalism [1] to derive the off-shell nilpotent and absolutely anticommuting (anti-)BRST symmetry transformations corresponding to the YM symmetries of the JP model. For this purpose, we first generalize the 3D basic fields to their corresponding superfields on the (3,2)-dimensional supermanifold parametrized by superspace variables $Z^{M}=\left(x^{\mu}, \theta, \bar{\theta}\right)$ where $x^{\mu}(\mu=0,1,2)$ are spacetime variables and $\theta, \bar{\theta}$ are Grassmannian variables (with $\theta^{2}=\bar{\theta}^{2}=0, \theta \bar{\theta}+\bar{\theta} \theta=0$ ). We also generalize the ordinary $3 \mathrm{D}$ exterior derivative $(d)$ to $(3,2)$-dimensional superexterior derivative $(\tilde{d})$. The explicit expressions are as follows:

$$
\begin{aligned}
& A_{\mu}(x) \longrightarrow \tilde{B}_{\mu}(x, \theta, \bar{\theta}), \quad C(x) \longrightarrow \tilde{F}(x, \theta, \bar{\theta}), \quad \bar{C}(x) \longrightarrow \tilde{\bar{F}}(x, \theta, \bar{\theta}), \\
& A^{(1)} \longrightarrow \tilde{A}^{(1)}=d Z^{M} \tilde{A}_{M} \equiv d x^{\mu} \tilde{B}_{\mu}(x, \theta, \bar{\theta})+d \theta \tilde{\bar{F}}(x, \theta, \bar{\theta})+d \bar{\theta} \tilde{F}(x, \theta, \bar{\theta}), \\
& d \longrightarrow \tilde{d}=d Z^{M} \partial_{M} \equiv d x^{\mu} \partial_{\mu}+d \theta \partial_{\theta}+d \bar{\theta} \partial_{\bar{\theta}} .
\end{aligned}
$$

Here, $\tilde{B}_{\mu}(x, \theta, \bar{\theta}), \tilde{F}(x, \theta, \bar{\theta})$ and $\tilde{\bar{F}}(x, \theta, \bar{\theta})$ are the superfields on the $(3,2)$-dimensional supermanifold and $\partial_{M}=\left(\partial_{\mu}, \partial_{\theta}, \partial_{\bar{\theta}}\right)$. In the second step, these superfields are expanded along Grassmannian direction $(\theta, \bar{\theta})$ as

$$
\begin{aligned}
\tilde{B}_{\mu}(x, \theta, \bar{\theta}) & =A_{\mu}(x)+\theta \bar{R}_{\mu}(x)+\bar{\theta} R_{\mu}(x)+i \theta \bar{\theta} S_{\mu}(x), \\
\tilde{F}(x, \theta, \bar{\theta}) & =C(x)+i \theta \bar{B}_{1}(x)+i \bar{\theta} B_{1}(x)+i \theta \bar{\theta} s(x), \\
\tilde{\bar{F}}(x, \theta, \bar{\theta}) & =\bar{C}(x)+i \theta \bar{B}_{2}(x)+i \bar{\theta} B_{2}(x)+i \theta \bar{\theta} \bar{s}(x),
\end{aligned}
$$

\footnotetext{
${ }^{2}$ Here we take the 3D flat Minkowski metric $\eta_{\mu \nu}=\operatorname{diag}(-1,+1,+1)$ and the Levi-Civita tensor follows $\varepsilon_{\mu \nu \eta} \varepsilon^{\mu \nu \eta}=-3$ !, $\varepsilon_{\mu \nu \eta} \varepsilon^{\mu \nu \sigma}=-2$ ! $\delta_{\eta}^{\sigma}$, etc., with $\varepsilon_{012}=+1=-\varepsilon^{012}$. We adopt dot and cross products $R \cdot S=R^{a} S^{a}, R \times S=f^{a b c} R^{a} S^{b} T^{c}$ in the $S U(N)$ Lie algebraic space spanned by the generators $T^{a}$ satisfying the algebra $\left[T^{a}, T^{b}\right]=f^{a b c} T^{c}$ with $a, b, c \ldots=1,2,3, \ldots, N^{2}-1$. The covariant derivative is defined as $D_{\mu} B^{a}=\partial_{\mu} B^{a}-g\left(A_{\mu} \times B\right)^{a}$.
} 
where, $R_{\mu}(x), \bar{R}_{\mu}(x), s(x), \bar{s}(x)$ are fermionic secondary fields and $S_{\mu}(x), B_{1}(x), \bar{B}_{1}(x), B_{2}(x)$, $\bar{B}_{2}(x)$ are bosonic in nature. Finally, we take the help of horizontality condition (HC) to determine the relationship amongst the basic and secondary fields of the theory.

We note that the kinetic term corresponding to the gauge field $A_{\mu}$ remains invariant under the gauge transformations (2). Thus, the HC implies that it should not be affected by the presence of Grassmannian variables when generalized onto the (3,2)-dimensional supermanifold. The above statement can be, mathematically, expressed as

$$
-\frac{1}{4} F^{\mu \nu} \cdot F_{\mu \nu}=-\frac{1}{4} \tilde{F}^{M N} \cdot \tilde{F}_{M N}
$$

where $\tilde{F}^{M N}$ is the super curvature defined on the $(3,2)$-dimensional supermanifold and can be derived from the Maurer-Cartan equation: $\tilde{F}^{(2)}=\tilde{d} \tilde{A}^{(1)}+i g\left(\tilde{A}^{(1)} \wedge \tilde{A}^{(1)}\right) \equiv \frac{1}{2 !}\left(d Z^{M} \wedge\right.$ $\left.d Z^{N}\right) \tilde{F}_{M N}$. The celebrated HC condition (6) leads to the following relationships amongst the basic, auxiliary and secondary fields

$$
\begin{aligned}
& R_{\mu}=D_{\mu} C, \quad \bar{R}_{\mu}=D_{\mu} \bar{C}, \quad B_{1}=-\frac{i}{2} g(C \times C), \quad \bar{B}_{2}=-\frac{i}{2} g(\bar{C} \times \bar{C}), \\
& S_{\mu}=D_{\mu} B+i g\left(D_{\mu} C \times \bar{C}\right) \equiv-D_{\mu} \bar{B}-i g\left(D_{\mu} \bar{C} \times C\right), \\
& s=-g(\bar{B} \times C), \quad \bar{s}=+g(B \times \bar{C}), \quad B+\bar{B}=-i g(C \times \bar{C}),
\end{aligned}
$$

where we have made the choices $\bar{B}_{1}=\bar{B}$ and $B_{2}=B$ which are, finally, identified with the Nakanishi-Lautrup type auxiliary fields. Substituting these relationships in the superexpansion (5), we have following explicit expansions:

$$
\begin{aligned}
\tilde{B}_{\mu}^{(h)}(x, \theta, \bar{\theta}) & =A_{\mu}(x)+\theta D_{\mu} \bar{C}(x)+\bar{\theta} D_{\mu} C(x)+\theta \bar{\theta}\left[i D_{\mu} B-g\left(D_{\mu} C \times \bar{C}\right)\right](x) \\
& \equiv A_{\mu}(x)+\theta\left(s_{a b} A_{\mu}(x)\right)+\bar{\theta}\left(s_{b} A_{\mu}(x)\right)+\theta \bar{\theta}\left(s_{b} s_{a b} A_{\mu}(x)\right), \\
\tilde{F}^{(h)}(x, \theta, \bar{\theta}) & =C(x)+\theta(i \bar{B}(x))+\bar{\theta}\left[\frac{g}{2}(C \times C)(x)\right]+\theta \bar{\theta}[-i g(\bar{B} \times C)(x)] \\
& \equiv C(x)+\theta\left(s_{a b} C(x)\right)+\bar{\theta}\left(s_{b} C(x)\right)+\theta \bar{\theta}\left(s_{b} s_{a b} C(x)\right), \\
\tilde{\bar{F}}^{(h)}(x, \theta, \bar{\theta}) & =\bar{C}(x)+\theta\left[\frac{g}{2}(\bar{C} \times \bar{C})(x)\right]+\bar{\theta}(i B(x))+\theta \bar{\theta}[(+i g(B \times \bar{C})(x)] \\
& \equiv \bar{C}(x)+\theta\left(s_{a b} \bar{C}(x)\right)+\bar{\theta}\left(s_{b} \bar{C}(x)\right)+\theta \bar{\theta}\left(s_{b} s_{a b} \bar{C}(x)\right),
\end{aligned}
$$

where $(h)$, as the superscript on the superfields, denotes the expansions of the superfields after the application of HC. Thus, we can read out the (anti-)BRST symmetry transformations $\left(s_{(a) b}\right)$ corresponding to the gauge field $A_{\mu}$ and (anti-)ghost fields $(\bar{C}) C$ from the above expressions. The (anti-)BRST symmetry transformations corresponding to the auxiliary fields $B$ and $\bar{B}$ can be obtained from the requirement of nilpotency and absolute anticommutativity properties of (anti-)BRST symmetries.

Furthermore, in order to derive the (anti-)BRST symmetry transformations for the vector field $\phi_{\mu}$ and the auxiliary field $\rho$, we have to go beyond the HC. For this purpose, we take help of gauge invariant restrictions (GIR) constituted with the help of composite fields $\left(F_{\mu \nu} \cdot \phi_{\eta}\right)$ and $\left(F_{\mu \nu} \cdot \rho\right)$ which remain invariant under gauge transformations (2). It is clear as below

$$
\delta_{1}\left(F_{\mu \nu} \cdot \phi_{\eta}\right)=0, \quad \delta_{1}\left(F_{\mu \nu} \cdot \rho\right)=0 .
$$

These gauge invariant quantities are physical ones (in some sense), thus, they must remain unaffected by the presence of Grassmannian variables when former quantities are generalized 
onto the $(3,2)$-dimensional supermanifold. Therefore, we have following GIR

$$
\begin{gathered}
\tilde{F}_{\mu \nu}^{(h)}(x, \theta, \bar{\theta}) \cdot \tilde{\phi}_{\eta}(x, \theta, \bar{\theta})=F_{\mu \nu}(x) \cdot \phi_{\eta}(x) \\
\tilde{F}_{\mu \nu}^{(h)}(x, \theta, \bar{\theta}) \cdot \tilde{\rho}(x, \theta, \bar{\theta})=F_{\mu \nu}(x) \cdot \rho(x) .
\end{gathered}
$$

In the above, $\tilde{\phi}_{\mu}(x, \theta, \bar{\theta})$ and $\tilde{\rho}(x, \theta, \bar{\theta})$ are superfields corresponding to the vector field $\phi_{\mu}(x)$ and $\rho(x)$, respectively, whereas $\tilde{F}_{\mu \nu}^{(h)}(x, \theta, \bar{\theta})$ is super 2-form curvature tensor. Now, following the same procedure as outlined above, we find the (anti-)BRST symmetry transformations corresponding to vector field $\phi_{\mu}$ and auxiliary field $\rho$. In explicit form, these (anti-)BRST symmetry transformations are

$$
\begin{array}{ll}
s_{a b} A_{\mu}=D_{\mu} \bar{C}, \quad s_{a b} \bar{C}=\frac{g}{2}(\bar{C} \times \bar{C}), & s_{a b} B=-g(B \times \bar{C}), \quad s_{a b} \bar{B}=0, \\
s_{a b} \phi_{\mu}=-g\left(\phi_{\mu} \times \bar{C}\right), \quad s_{a b} C=i \bar{B}, \quad s_{a b} \rho=-g(\rho \times \bar{C}), & \\
s_{b} A_{\mu}=D_{\mu} C, \quad s_{b} C=\frac{g}{2}(C \times C), & s_{b} \bar{B}=-g(\bar{B} \times C), \quad s_{b} B=0, \\
s_{b} \phi_{\mu}=-g\left(\phi_{\mu} \times C\right), \quad s_{b} \bar{C}=i B, \quad s_{b} \rho=-g(\rho \times C) .
\end{array}
$$

Furthermore, it can be checked that the above mentioned (anti-)BRST symmetry transformations are off-shell nilpotent (i.e. $\left.s_{(a) b}^{2}=0\right)$ and absolutely anticommunting (i.e. $s_{b} s_{a b}+s_{a b} s_{b}=$ $0)$ in nature in their operator form.

\section{Curci-Ferrari Restriction}

A close look at (7) reveals that the Curci-Ferrari restriction $[B+\bar{B}=-i g(C \times \bar{C})]$ is a natural outcome of superfield approach. Actually, this condition arises when we set $\tilde{F}_{\theta \bar{\theta}}$ component of supercurvature tensor to be zero. It connects the Nakanishi-Lautrup auxiliary fields $B$ and $\bar{B}$ with the (anti-)ghost fields $(\bar{C}) C$ of the theory. The CF restriction is a hallmark of any non-Abelian 1-form gauge theory [12] and plays a central role in providing the proof for absolute anticommutativity of (anti-)BRST symmetry transformations. It also plays an important role in obtaining a set of coupled Lagrangian densities which respect the above derived (anti-)BRST symmetry transformations (11). The details may be found in Ref. [8, 9].

\section{Conclusions}

In this talk, we summarize our results on the $3 D$ massive Jackiw-Pi model. We have derived (anti-)BRST symmetry transformations corresponding to the YM symmetries of JP model. One of the novel features of this investigation is the derivation of (anti-)BRST transformations for the auxiliary field $\rho$ from our superfield formalism which is neither generated by the (anti-)BRST charges nor obtained from the requirements of nilpotency and/or absolute anticommutativity of the (anti-)BRST symmetries for our 3D model. The Curci-Ferrari restriction, which plays a central role in providing the proof for absolute anticommutativity of (anti-)BRST symmetry transformations, is a natural outcome of this superfield approach.

Acknowledgments: Financial support from The Institute of Mathematical Sciences, Chennai, India is gratefully acknowledged. The author would also like to thankfully acknowledge his collaborators, R. P. Malik and R. Kumar, with whom the present work is completed. 


\section{References}

[1] Schwinger J.

Gauge invariance and mass

Phys. Rev. 1962, 125, 397-398.

[2] Freedman D. Z., Townsend P. K.

Antisymmetric tensor gauge theories and non-linear $\sigma$-models

Nucl. Phys. B 1981, 177, 282-296.

[3] Allen T. J., Bowick M. J., Lahiri A.

Topological mass generation in $3+1$ dimensions

Mod. Phys. Lett. A 1991, 6, 559-571.

[4] Gupta S., Kumar R., Malik R. P.

Absolutely anticommuting (anti-)BRST symmetry transformations for topologically massive Abelian gauge theory

Eur. Phys. J. C 2010, 70, 491-502.

[5] Deser S., Jackiw R., Templeton S.

Three-dimensional massive gauge theories

Phys. Rev. Lett. 1982, 48, 975-978.

[6] Dayi Ö. F.

Hamiltonian formulation of Jackiw-Pi three-dimensional gauge theories

Mod. Phys. Lett. A 1998, 13, 1969-1977.

[7] Cima O. M. Del

The Jackiw-Pi model and its symmetries

J. Phys. A: Math. Theor 2011, 44, 352001.

[8] Gupta S., Kumar R., Malik R. P.

Nilpotent symmetries in the Jackiw-Pi model of 3D massive non-Abelian theory: superfield approach

arXiv:1108.1547 [hep-th].

[9] Gupta S., Kumar R.

Augmented superfield approach to non-Yang-Mills symmetries of Jackiw-Pi model: novel observations

Mod. Phys. Lett. A 2013, 28, 1350011.

[10] Batalin I. A., Lavrov P. M., Tyutin I. V.

Covariant quantization of gauge theories in the framework of extended BRST symmetry J. Math. Phys. 1990, 31, 1487-1493.

[11] Bonora L., Tonin M.

Superfield formulation of extended BRS symmetry

Phys. Lett. B 1981, 98, 48-50. 
[12] Curci G., Ferrari R.

Slavnov transformations and supersummetry

Phys. Lett. B 1976, 63, 91-94. 